\title{
Transverse Envelope Dynamics of a 28.5 GeV Electron Beam in a Long Plasma
}

\author{
C.E. Clayton, B.E. Blue, E.S. Dodd, C. Joshi, K.A. Marsh, W.B. Mori, S. Wang \\ University of California, Los Angeles, CA 90095 USA \\ P. Catravas, S. Chattopadhyay, E. Esarey, W.P. Leemans \\ Lawrence Berkeley National Laboratory, University of California, Berkeley CA 94720 USA \\ R. Assmann*, F.J. Decker, M.J. Hogan, R. Iverson, P. Raimondi, R.H. Siemann, D. Walz \\ Stanford Linear Accelerator Center, Stanford University, Stanford CA 94309 USA \\ T. Katsouleas, S. Lee, P. Muggli ${ }^{\dagger}$ \\ University of Southern California, Los Angeles, CA 90089 USA
}

(October 9, 2001)

\begin{abstract}
The transverse dynamics of a $28.5 \mathrm{GeV}$ electron beam propagating in a $1.4 \mathrm{~m}$ long, $0-2 \times 10^{14} \mathrm{~cm}^{-3}$ plasma are studied experimentally in the underdense or blow-out regime. The transverse component of the wake field excited by the short electron bunch focuses the bunch, which experiences multiple betatron oscillations as the plasma density is increased. The spot size variations are observed using optical transition radiation and Cherenkov radiation. In this regime, the behavior of the spot size as a function of the plasma density is well described by a simple beam envelope model. Dynamic changes of the beam envelope are observed by time resolving the Cherenkov light.

PACS numbers: 29.27.-a, 41.85.-p, 41.75.Lx, 52.38.-r
\end{abstract}

Particle acceleration by space charge waves in plasmas is attractive because such waves can have accelerating gradients that are orders of magnitude larger than those found in conventional radio-frequency structures [1]. While laser-driven plasma waves have realized accelerating gradients of greater than $100 \mathrm{GeV} / \mathrm{m}$, the acceleration length has been limited to about a millimeter [2]. On the other hand, electron beam-driven plasma accelerators have produced plasma waves over a length of about $12 \mathrm{~cm}$, but the gradients have been limited to about $25 \mathrm{MeV} / \mathrm{m} \mathrm{[3].} \mathrm{In} \mathrm{the} \mathrm{experiment} \mathrm{now} \mathrm{being} \mathrm{con-}$ ducted at the Stanford Linear Accelerator Center a prototype plasma accelerator stage that would demonstrate high-gradient acceleration over a distance of greater than a meter is being developed. It requires the stable propagation of high current $(>1 k A)$, very short (few ps) drive electron bunches through dense $\left(>10^{14} \mathrm{~cm}^{-3}\right)$ plasmas. The propagation of such bunches through a long, dense column of plasma has certain time dependent and plasma density dependent transverse behavior that can be theoretically predicted. In this Letter the predicted phenomena are demonstrated experimentally; namely multiple oscillations of the beam spot size as a result of betatron motions, and time dependent variation of the beam envelope because of finite rise time of the beam and response time of the plasma electrons.

Previous experiments on the focusing of electron beams in plasmas have shown good agreement with envelope

\footnotetext{
*Present address: CERN, Geneva, Switzerland

${ }^{\dagger}$ Corresponding author: muggli@usc.edu
}

models in the overdense regime where the plasma density is larger than the beam density [5], and in the return current cancellation regime [4] where the transverse spot size of the electron beam is comparable to the plasma skin depth, $c / \omega_{p}$. This Letter presents the first experimental study of mono-energetic beam propagation in long dense plasmas in the underdense or blow-out regime where the beam density is larger than the plasma density; conditions that are relevant to future high energy plasma accelerators. In the plasma wakefield accelerator the plasma wave, or wake, is driven by a short, relativistic electron bunch. The plasma wake has longitudinal accelerating fields and transverse focusing fields. The transverse component of the wake focuses the beam which can overshoot, and undergo multiple betatron oscillations over the plasma length.

The head of a round bunch of radius $\sigma_{r}$, length $\sigma_{z}$, and with with $\mathrm{N}$ electrons, with a density $n_{b}=$ $N /(2 \pi)^{3 / 2} \sigma_{r}^{2} \sigma_{z}$ larger than the plasma density $n_{p}$ expels all the plasma electrons to a radius $r_{c}=$ $\left(N /(2 \pi)^{3 / 2} \sigma_{z} n_{p}\right)^{1 / 2}$, where $\alpha=1$ for a long bunch and $\alpha=2$ for a short bunch of the order of a plasma wavelength long. In this underdense or blow-out regime, the focusing strength of the pure ion column $S$ is that of a uniform cylinder of charge density $n_{p}$ [6]: $S=n_{p} e / 2 c \epsilon_{0}$. It is constant over much of the bunch length, and is constant over the radius of the ion column, which acts as a plasma lens free of geometrical aberrations. The behavior of the electron beam with normalized emittance $\epsilon_{N}$ is described by the beam envelope equation [7]:

$$
\frac{d^{2} \sigma_{r}(z)}{d z^{2}}+\left[K^{2}-\frac{\epsilon_{N}^{2}}{\gamma^{2} \sigma_{r}^{4}(z)}\right] \sigma_{r}(z)=0
$$


where $K=\left(e S / \gamma m_{e} c\right)^{1 / 2}=\omega_{p} /(2 \gamma)^{1 / 2} c$ is the plasma restoring constant, $\omega_{p}=\left(n_{p} e^{2} / \epsilon_{0} m_{e}\right)^{1 / 2}$ is the electron plasma frequency, $\gamma$ is the beam relativistic factor, and $\mathrm{c}$ is the speed of light in vacuum. The beam envelope evolution before and after the plasma is described by Eq. (1) with $K=0$. Note that energy loss or gain by the particles is not included in this simple model.

The solution to Eq. (1) for an unmatched beam, $\beta_{\text {beam }}=\gamma \sigma_{r}^{2} / \epsilon_{N} \neq 1 / K=\beta_{\text {plasma }}$, is well known and describes the oscillation of the beam envelope with a spatial period $\lambda_{\beta}=\pi c(2 \gamma)^{1 / 2} / \omega_{p}$ equal to half the betatron wavelength of the beam particles. The phase advance experienced by the beam over the plasma length is $\Psi_{L}\left(n_{p}\right)=\int_{0}^{L} d z / \beta_{\text {plasma }}=\pi L / \lambda_{\beta} \sim n_{p}^{1 / 2} L$, and can amount to multiples of $\pi$ in a long, dense plasma.

The simple results presented above provide guidance for interpreting the wake fields obtained from fully relativistic, 3-dimensional particle in cell simulations [8]. The transverse component of the field obtained from these simulations is shown in Fig. 1 for two beam radii. For both radii, $n_{b}>n_{p}$. The electrons in the head of the bunch expel the plasma electrons, and the transverse focusing strength increases along the bunch. Figure 1 shows that for a given plasma density, the blow-out occurs sooner for a larger bunch density (smaller $\sigma_{r}$ ). In the case where $\beta_{\text {beam }}>\beta_{\text {plasma }}$, the blow-out is reached earlier in the bunch as the bunch is focused within the plasma. Numerical simulations show that the time to reach blow-out depends on the beam density and not on the plasma density. Near the tail of the bunch, a defocusing field region exists where the plasma electrons rush back to the axis $\left(\approx \pi / \omega_{p}\right.$ in the bunch, Fig. 1$)$.

The experimental set-up [9] is shown in Fig. 2. In the experiment performed at the Stanford Linear Accelerator Center the $28.5 \mathrm{GeV}$ electron beam from the main linac is transported through the Final Focus Test Beam line to a $1.4 m$ long plasma with a density in the $0-2 \times 10^{14} \mathrm{~cm}^{-3}$ range. The plasma is produced by single-photon ionization of a lithium vapor contained in a heat-pipe oven [10] [11], by an ultraviolet laser pulse. The initial plasma density (at the time the laser fires) is obtained from the number of absorbed photons and the volume of the lithium vapor intercepted by the laser beam. The plasma density is allowed to decay for a few miccoseconds by recombination and diffusion to obtain a more uniform plasma. The typical time for the plasma density to decrease by a factor of two is about $12 \mu \mathrm{s}$ [11]. The plasma density at the time the electron beam traverses the plasma is obtained from the plasma initial density and decay rate, from the minima of the beam spot size observed downstream from the plasma as a function of the uv laser pulse energy (Fig.3), and from the Cherenkov radiation emitted by the electron beam in the lithium vapor. It was previously shown that the values of np obtained simultaneously using the two last methods are in good agreement
[12]. The typical electron beam and plasma parameters are given in Table I. Note that $n_{b}>n_{p}$ for the entire range of experimental parameters. The size of the beam is monitored by imaging the Optical Transition Radiation (OTR) emitted when traversing thin titanium foils located $\approx 1 m$ upstream and $\approx 1 m$ downstream from the plasma. The spatial resolution of the $1: 1$ imaging system is $\approx 20 \mu \mathrm{m}$. After exiting the plasma, the electron beam travels through a dipole magnet and a thin piece of aerogel located $\approx 12 \mathrm{~m}$ from the plasma (Fig. 2). The Cherenkov light emitted from the aerogel is imaged onto a charge coupled device camera, and onto the slit of a streak camera to obtain the time-integrated and the time resolved spot size of each bunch, with a time resolution of $\approx 1 p s$ and a spatial resolution of $\approx 100 \mu \mathrm{m}$.

Figure 3 shows the horizontal $\left(\sigma_{x}\right)$ and vertical $\left(\sigma_{y}\right)$ beam spot sizes measured at the downstream OTR location as functions of the plasma density expressed as the phase advance $\Psi_{L}$ of the bunch particles over the plasma length. Note that the electron beam parameters are different in both planes. In the case of Fig. 3 the plasma density is varied by changing the ionizing laser pulse energy. The maximum value of $n_{p}$ is $\approx 1.8 \times 10^{14}$ at $\Psi_{L} \approx 3.4 \pi$. The beam spot sizes $\sigma_{x}$ and $\sigma_{y}$ are defined as the root-mean-square of gaussian fits to the OTR images summed in the vertical and horizontal directions, respectively. The first minimum $\left(\Psi_{L} \approx 0.5 \pi\right.$ on Fig. 3 a and b) corresponds to the plasma acting as an extended lens [13], and the reduction in spot size at that location is roughly a factor of two in both planes compared to a typical shot to shot variations of $\pm 10 \%$ in both planes for the incoming beam (upstream OTR location). The beam spot size modulation observed when increasing the plasma density is the result of the multiple minima of the beam envelope within the plasma.

In order to compare the experimental data to the envelope model predictions, the beam emittance, initial spot size and convergence angle at the plasma entrance (or equivalently, the beam Courant-Snyder parameters) need to be known. Although the electron beam parameters of the Final Focus Test Beam are well known, scattering of the beam when traversing the beryllium window $(75 \mu \mathrm{m}$ thick), the titanium OTR foil $(36 \mu \mathrm{m})$, and the $\mathrm{SiO}_{2}$ pellicle $(230 \mu \mathrm{m})$ located before the plasma increases the beam emittance and changes the waist location as compared to vacuum propagation values. The $\mathrm{SiO}_{2}$ pellicle used to make the ionizing laser pulse colinear with the electron beam is responsible for most of the beam emittance growth. It accounts for normalized emittance growths [14] of $\epsilon_{N, \text { scatt }, x} \approx 4.1 \times 10^{-5} \mathrm{~m}-\mathrm{rad}$ and $\epsilon_{N, \text { scatt }, y} \approx 2.1 \times 10^{-5} \mathrm{~m}-\mathrm{rad}$ to be added in quadrature to the emittance in the horizontal and vertical plane, respectively. The beam parameters after the scattering elements, but before the plasma entrance, are obtained by fitting solutions to the envelope equation (Eq. 1) to the measured spot sizes at three locations with the 
plasma off. The solutions to the envelope equation (Eq. 1) for a beam with a waist at the $\mathrm{SiO}_{2}$ pellicle, and with the parameters determined by the fit are also shown on Figs. $3 \mathrm{a}$ and $\mathrm{b}$. The agreement between the values of $\sigma_{x, y}$ derived from Eq. 1 and the values measured in the experiment is very good in the horizontal plane (Fig. $3 \mathrm{a}$ ), and reasonable in the vertical plane (Fig. $3 \mathrm{~b}$ ), even though the model does not include possible spherical aberrations due to plasma inhomogeneities, or longitudinal aberrations due to the head to tail increase of the focusing force. However, Figure 1 shows that in the case of the $35 \mu \mathrm{m}$ beam radius, more than $75 \%$ of the charge of the beam experiences a focusing strength larger than $90 \%$ of the blow-out value. The effect of the spot size variations along the bunch is thus expected to be a small contribution to the measurement of the time integrated spot sizes (Fig. 3). Other possible effects that could lead to a difference between the measured spot sizes and those predicted by the simple model include the presence and oscillation of a beam tail, and the effect of the defocusing field in the tail region of the beam. As shown in Fig. 1, because of the inertia of the plasma electrons the electron beam experiences a focusing strength that varies along the bunch from zero to that of a pure ion channel. Therefore different time slices of the beam can experience different number of betatron oscillations over the length of the plasma. In order to investigate this dynamic change in beam envelope experimentally, the beam spot size is made larger $\left(\sigma_{x} \approx \sigma_{y} \approx 75 \mu \mathrm{m}\right)$, causing the blow-out to be reached later in the bunch. The dynamic range and temporal resolution of the streak camera as well as the vertical dispersion place a limit on the temporal and spatial features observed in the experiment. In the absence of plasma the beam radius in the vertical plane at the Cherenkov radiator location (Fig. 2) is $\sigma_{y} \approx 600 \mu \mathrm{m}$, and is constant along the bunch as can be seen in Fig. 4 a and d. At that location the dispersion $\eta$ is $10 \mathrm{~cm}$. The typical beam energy spread is $0.3 \%$, thus corresponding to an equivalent spot size of $\approx 300 \mu \mathrm{m}$ to be added in quadrature to the spot sizes obtained from the beam envelope model. Evidence of dynamic changes in the beam envelope is seen on a typical streak camera image (Fig. $4 \mathrm{~d}$ and e). With a plasma density of $\approx 0.2 \times 10^{14} \mathrm{~cm}^{-3}$ or $\Psi_{L} \approx 1.2 \pi$, the streak camera image shows defocusing of the head of the bunch, followed by a refocusing of the bunch to approximately its original size. Note that at this low plasma density very small energy loss and no energy gain are expected.

A beam envelope model described above is applied with the time-dependent transverse fields to describe the features of the single bunch dynamic focusing shown in Fig. 4. The focusing force experienced by each individual beam slice is obtained from the transverse field derived from the numerical simulations (Fig. 1) for the relevant bunch parameters. Each slice is propagated in the plasma and in vacuum using Eq. 1, and the time resolved beam envelope reconstructed. The result of this procedure with the same parameters as those of the experiment is shown in Fig. $4 \mathrm{c}$ and $\mathrm{f}$. Figure $4 \mathrm{f}$ shows that as the focusing strength increases along a single bunch, different slices of the beam experience a different number of envelope oscillations. There is a reasonable qualitative agreement between the experimental result (Fig. $4 \mathrm{~d}$ and e) and the model (Fig. $4 \mathrm{f}$ ).

In summary, the propagation of a $28.5 \mathrm{GeV}$ electron beam in a $1.4 \mathrm{~m}$ plasma has been demonstrated. The beam density is larger than the plasma density, and the beam is not matched to the plasma. The beam envelope undergoes multiple oscillations over the plasma length. The corresponding variations of the beam spot size with the plasma density are clearly observed experimentally. The transverse dynamics of the beam in the underdense or blow-out regime is well described by considering the action of an ideal ion column on the electron beam envelope. The time dependent transverse dynamics associated with the plasma channel formation by the bunch head is observed with a large radius electron beam in a low density plasma. The experimental observation of the beam transverse dynamics is a direct evidence of the excitation of the large amplitude transverse plasma wake and implies that a loss of energy by the particles in the core of the electron bunch has occurred. This will be the subject of a future publication.

This work is supported by the US DoE Grants No. DEFG03-92ER40745, No. DE-AC03-76SF00515, No. DEFG03-98DP00211, and No. DE-FG03-92ER40727, and NSF grants No. ECS-9632735 and No. DMS-9722121. We would like to thank Dr. Peter Tsou of JPL for providing the aerogel.

[1] C.Joshi et al., Nature 311, 525 (1984).

[2] A. Modena et al. Nature 37, 606 (1995), C.I. Moore et al. Phys. Rev. Lett. 79, 3909 (1997), R. Wagner et al. Phys. Rev. Lett. 78, 3125 (1997), D.F. Gordon et al. Phys. Rev. Lett. 80, 2133 (1998).

[3] N. Barov et al., PRST-AB 3, 011301, (2000).

[4] J.B. Rosenzweig et al. Phys. Fluids B 2, 1376 (1990), Nakanishi et al. Phys. Rev. Lett. 66, 1870 (1991), and G. Hairapetian et al. Phys. Rev. Lett. 72, 2403 (1991).

[5] R. Govil et al. Phys. Rev. Lett. 83, 3202 (1555).

[6] S. Lee et al. Phys. Rev. E 61(6), 7014 (2000).

[7] K.G. Steffen, in High Energy Beam Optics, Interscience: John Wiley, New York, 173 (1965).

[8] R.G. Hemker et al. Proceedings of the 1999 Particle Accelerator Conference, 3672 (1999).

[9] M. Hogan et al. Phys. Plasmas 7, 2241, (2000).

[10] C.R. Vidal and J. Cooper, J. Appl. Phys. 40(8), 3370 (1960). 
[11] P. Muggli et al. IEEE Trans. On Plasma Science, 27(3), 791 (1999).

[12] P. Catravas et al. Phys. Rev. E, 64, 046502 (2001).

[13] P. Chen, Part. Accel. 20, 171 (1985).

[14] D.C. Carey, "The Optics of Charged Particle Beams," Harwood Academic Publishers (1992).

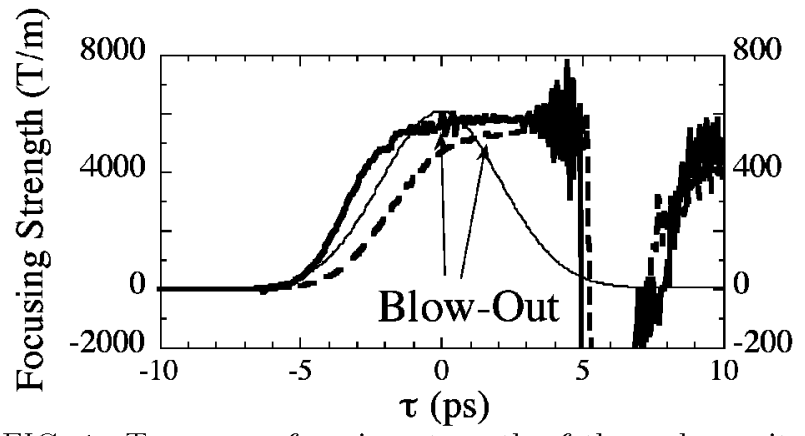

FIG. 1. Transverse focusing strength of the wake excited by an electron bunch (thin line) with the parameters of Table Table1 with $\sigma_{r}=35 \mu \mathrm{m}$ (continuous line) and $\sigma_{r}=70 \mu \mathrm{m}$ (dashed line) in a plasma with $n_{p}=2 \times 10^{14} \mathrm{~cm}^{-3}$ as obtained from PIC numerical simulations. The peak defocusing field strength is greater than $-16000 T / m$ at $\approx 5.5 p s$ (not visible).

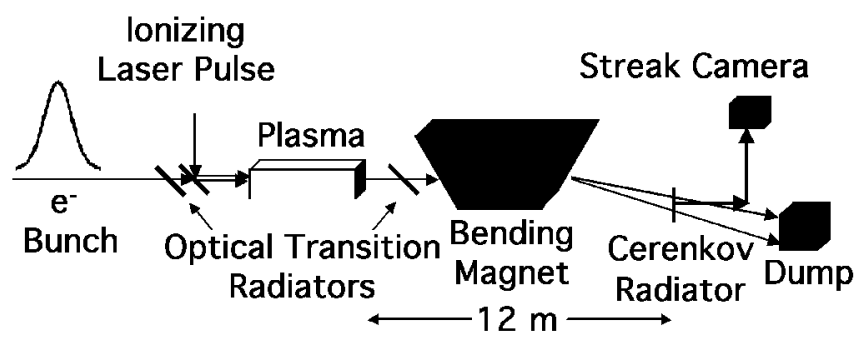

FIG. 2. Schematic of the experimental set-up. Not shown are two $75 \mathrm{~m}$ beryllium windows (located between the upstream OTR foil and the $\mathrm{SiO}_{2}$ pellicle, and after the downstream OTR foil) that isolate the linac vacuum from the plasma chamber.

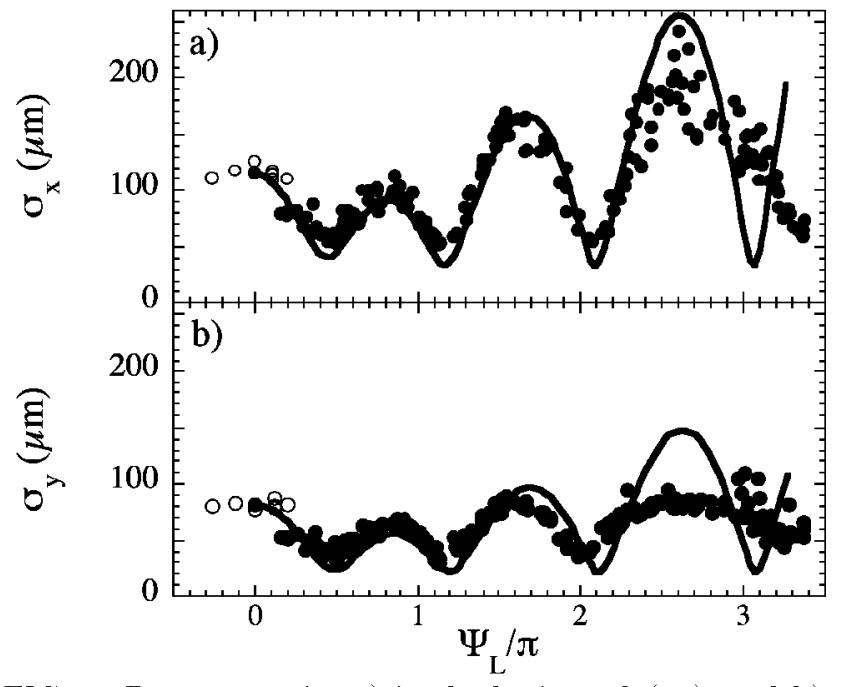

FIG. 3. Beam spot size a) in the horizontal $\left(\sigma_{x}\right)$, and b) in the vertical plane $\left(\sigma_{y}\right)$ measured at the downstream OTR location as a function of the plasma density expressed as the phase advance $\Psi_{L} \sim n_{p}^{1 / 2} L$ of the beam in the plasma normalized to $\pi$. Each symbol corresponds to a measurement of a single event. The empty circles correspond to beam propagation in vacuum $\left(n_{p}=0\right.$, ionization laser off $)$. The solid lines are the best fits of the solutions to the envelope equation (Eq. 1) obtained for a beam with a waist at the $\mathrm{SiO}_{2}$ pellicle, and with: a) $\sigma_{x 0}=39 \mu \mathrm{m}, \epsilon_{N, x}=8 \times 10^{-5} \mathrm{~m}-\mathrm{rad}$ $\left(\beta_{x 0}=1.06 \mathrm{~m}\right)$, and b) $\sigma_{y 0}=20 \mu \mathrm{m}, \epsilon_{N, y}=3 \times 10^{-5} \mathrm{~m}-\mathrm{rad}$ $\left(\beta_{y 0}=0.73 m\right)$.
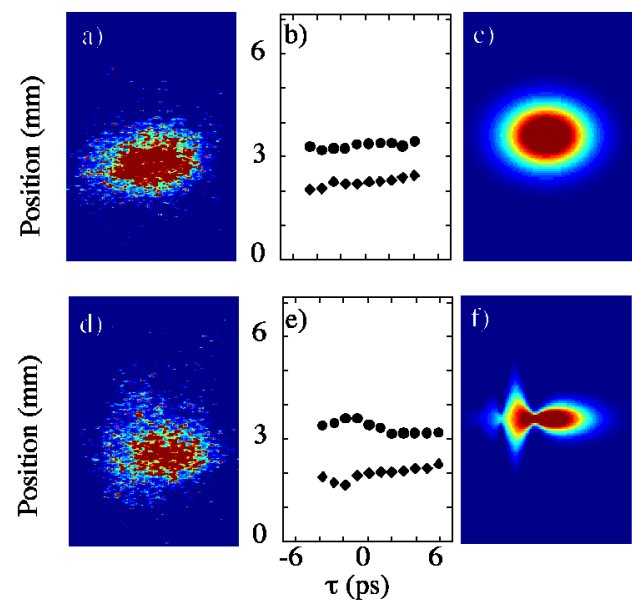
FIG. 4. Time resolved streak camera images of the bunch (single event) in the y-plane, $\approx 12 m$ downstream from the plasma, with a) plasma off $\left(n_{p}=0\right)$, and d) plasma on $\left(n_{p}=0.24 \times 10^{14} \mathrm{~cm}^{-3}\right)$. Figures b) and e) show the $\pm \sigma_{y}$ locations (circles and diamonds respectively) of $1 p s$ time slices, obtained from gaussian fits to Figures a and d . Figures d and e show an overall defocusing of the beam head, while the core of the beam is refocused to about its size in absence of plasma. Figures $\mathrm{c}$ and $\mathrm{f}$ show the calculated bunch size after propagation through the plasma and $12 \mathrm{~m}$ in vacuum, using the focusing strength of Fig. 1 and Eq. 1 for each slice along the bunch. The beam parameters are those of Table Table1, with $\sigma_{r}=75 \mu \mathrm{m}$. Additional scattering from a pellicle and foils located after the plasma contribute to the observed spot size.

\begin{tabular}{|l|c|c|}
\hline \hline \hline Number of $e^{-}$per bunch & $N$ & $2 \times 10^{10}$ \\
\hline Bunch Energy & $E, \gamma$ & $28.5 \mathrm{GeV}, 5.6 \times 10^{3}$ \\
\hline Bunch Radius $(\mathrm{rms})$ & $\sigma_{x}, \sigma_{y}$ & $35 \mu \mathrm{m}$ \\
\hline Bunch Length $(\mathrm{rms})$ & $\sigma_{z}$ & $0.6 \mathrm{~mm}$ \\
\hline Normalized Emittances & $\epsilon_{x}, \epsilon_{y}$ & $5,0.5 \times 10_{-5}(\mathrm{~m}-\mathrm{rad})$ \\
\hline Plasma Density & $n_{p}$ & $0-2 \times 10_{14}\left(\mathrm{~cm}^{-3}\right)$ \\
\hline Plasma Length & $L$ & $1.4(\mathrm{~m})$ \\
\hline \hline
\end{tabular}

TABLE I. Typical beam and plasma parameters 